\title{
Constraints and Effects of Partner Selection on the Emergence of Structures in Social Environments
}

\author{
Elpida S. Tzafestas \\ Cognitive Science Laboratory \\ Department of Philosophy and History of Science \\ University of Athens \\ University Campus, Ano Ilisia 15771, Athens, GREECE. \\ etzafestas@phs.uoa.gr
}

\begin{abstract}
In this work, we are exploring the internals and effects of partner selection on spatial and social dynamics and clashes. We are considering two different social settings: (a) one involving imitation dynamics that is exemplified by a modified Axelrod cultural simulation model extended with a Moore neighborhood, heterogeneous sets of cultural features per agent and a number of psychologically realistic, basic and more advanced, conceptual models of cultural affinity perception and imitation, and (b) one involving game-like interaction dynamics that is exemplified by a model of social noisy IPD interaction with an additional attraction mechanism that makes agents unconditionally cooperative toward attractive opponents. In both models, a simple mechanism of partner selection has been found to modify the social environment by allowing different types of social structures to emerge, such as fast built cultural homogeneous groups in the case of cultural simulation or groups of interacting cooperative agents that are attracted by one another in the case of IPD with attraction. We are identifying a number of cognitive factors that are used to model partner selection, namely memory depth, learning speed and openness, and we study the phenomena obtained. We are finally briefly discussing how these factors may be studied and taken into account when designing complex sociotechnical systems.
\end{abstract}

Keywords-component; Social simulation; Cultural imitation; IPD; Attraction; Social clash; Social network; Partner selection.

\section{INTRODUCTION}

Social simulation is the research area that uses computational and modeling techniques to study social phenomena and systems, ranging from political science to sociology, linguistics and even sometimes social psychology. The usual method employed is the modeling and simulation of a society of agents, each one having its own view of the world, as well as its own goals, roles and capabilities. Behavioral interaction within such a social environment means that the agents will meet and interact with often very different agents of conflicting viewpoints and goals. Regardless of the model of interaction adopted (flat spaceless, spatially defined within a delimited environment or through fixed or dynamic network connections), an agent will profit if it may select its partners so as to individually optimize its own results. The partner selection process has been partly inspired by sexual selection introduced in the context of the Darwinian theory, where an organism selects its mating partners so as to maximize its reproductive success that shows in the number of its offspring. This evolutionary paradigm has been adopted by evolutionary and behavioral economics which constitute traditionally one of the pioneered domains of research in agent-based and social simulation, often with the aid of game theoretic methods and terms. For this reason, partner selection is well-established as the phenomenon where an agent selects the agents it will interact with so as to maximize its personal scores or payoffs (see for example [1]).

In this paper we are examining the effects of partner selection on two exemplary social simulation cases and investigate its internal cognitive parameters that act as constraints to the process. Section 2 presents a fundamental partner selection algorithm that is used in the exemplary cases described in fair detail in sections 3 and 4 . In section 5, the algorithm is dissected to study the parameters of cognitive nature that influence the outcome of partner selection in various environments. Finally, section 6 discusses the implications of the findings of previous sections and concludes.

\section{BASICS OF PARTNER SELECTION}

Partner selection as used by an agent in social simulations is a means to constrain the set of partners it interacts with to a few agents that allow a high interaction payoff. In the following paragraphs we present the results of comparative experiments with and without partner selection to understand the effect of partner selection and identify the parameters involved. Partner selection uses a simple probabilistic preference scheme, previously introduced in [2], where each agent maintains a set of probabilities of interaction with each of the other agents it may interact with. All partners are equiprobable in the beginning and the corresponding preferences develop after each interaction according to the following general reinforcement algorithm:

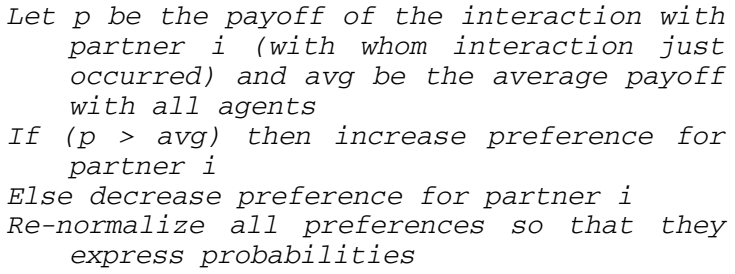

The algorithm may be instantiated in various social interaction contexts. We have selected two paradigmatic 
social interaction cases, namely one where interaction is imitation-like, and one where interaction is game-like. In the first case, the result of any interaction is "inherited" by the subsequent interactions, in the sense that an imitation interaction results in internal change in at least one of the agents and this change will become manifest in subsequent interactions (the imitation potential of the considered agent will be different in the next round ). In the second case, the result of an interaction is not inherited, but the "score" obtained between any pair agents is independent of the one between any other pair. Thus the relation between any two agents is nowhere close to being partly "transitive" as in the imitation case (there, if agent $\mathrm{A}$ resembles agent $\mathrm{B}$ and agent $B$ resembles agent $C$, then agent $A$ resembles agent $C$, with all resemblance relations being partial).

\section{CULTURAL IMITATION}

\section{A. Rationale and Model}

With this model, we are exploring spatial dynamics and clashes in cultural simulations involving multi-cultural populations with partner selection. We are using as basis an Axelrod model of cultural imitation [3][4][5][6], where an agent is characterized by a number of cultural features, each one of which may take one of several values, called traits, and where an initially fully heterogeneous population (with random traits) may lead eventually to compact cultural groups that are homogeneous internally but with large differences between them, provided that a simple imitation process takes place: an agent may imitate (copy) a trait of a neighbor probabilistically based on the affinity with him (the proportion of traits that coincide). We have modified and extended this model with a Moore neighborhood, heterogeneous sets of cultural features per agent and a number of psychologically realistic, basic and more advanced, conceptual models of cultural affinity perception and imitation [7]. We have shown that in many cases the population stabilizes to multi-cultural configurations and that, in cases of population clashes where two or more culturally contiguous populations meet, the cultural divide may persist, albeit in a relatively weaker form [7][8]. In this paper we repeat some of our previous experiments to investigate the effects of partner selection on these configurations.

First, we are briefly summarizing the extended models that we are using (for an in-depth account, see [7]). Our models have been inspired by the observation (also put forward by other authors, for instance [6]) that the original Axelrod result, where cultural grouping and polarization emerges in an initially diverse society, is a combined sideeffect of the model assumptions of 4-connectivity and fewer features than traits per feature. If instead, more features are used with fewer traits each, all systems eventually lead to monoculture. On top of this, if 8-connectivity (Moore neighborhood) is assumed, systems converge to full affinity substantially faster. It is this modeling intricacy that led us to reflect initially on the factors that may be responsible for the emergence of diverse cultural groups.
The heterogeneous Axelrod model is an Axelrod model, where each agent may have a variable number of cultural features, that are initially ordered. For example, agent-i may have (the first) five features while agent-j may have (the first) nine features etc. This way, some features are widespread within the population and others are not. A number of individualistic models have been defined: selfish models where agents have feature vectors where some features may be absent and the computation of affinity uses only common features, indifferent models where agents have the full set of features but during affinity computation they may be indifferent to some of them, models with individualbased adaptation of the above feature vectors and others (see [7]).-Some selfish models have been found to yield lower perceived than actual affinities, i.e. different affinities as perceived individually than as perceived from the outside, while some indifferent models have been found to yield lower actual than perceived affinities, that is the agents arrive to a happy stable state that looks very diverse from the outside. Finally, a model called "complex" uses degrees of indifference toward cultural features. Each cultural feature is assigned a real-valued weight between 0 and 1: the lower its value, the more indifference the agent will show toward the feature. The perceived affinity of an agent with another one is defined accordingly as $\Sigma \mathrm{w}_{\mathrm{i}} \mathrm{aff}_{\mathrm{i}} / \Sigma \mathrm{w}_{\mathrm{i}}$, while the actual affinity is defined as usually. Complex models lead to very diverse but stable systems with almost full affinity in common features but individually perceived affinities that take a broad range of values, because agents tend to perceive each other very differently due to their different weight vectors

\section{B. Experiments}

We have performed a series of experiments with and without partner selection. Partner selection uses the algorithm of section 2, instantiated as follows (the exact details of instantiation are highlighted):

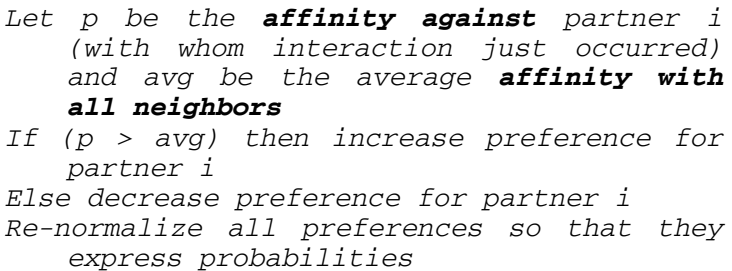

Unless otherwise stated, all experiments that follow use a $10 \times 10$ world, up to 15 cultural features of up to 10 traits each and a partner set $\mathrm{K}=3$ agents, that is, in each round an agent uses up to 3 agents to interact with and updates its preferences according to the results of the interaction.

A simple initial experiment concerns a modified homogeneous Axelrod model with an initially diverse population (fig. 1). Partner selection does not change the final outcome which is full monoculture in the population, but it substantially speeds up the cultural contagion process. On the other hand, partner selection induces extremely fast local polarization leading to intermediate configurations that are not possible without partner selection in this model. Fig. 1 shows the large speed of local convergence throughout the 
experiment. It also shows the convergence of the "preferred" affinity, i.e. the average affinity with neighbors weighted by the preference factors.

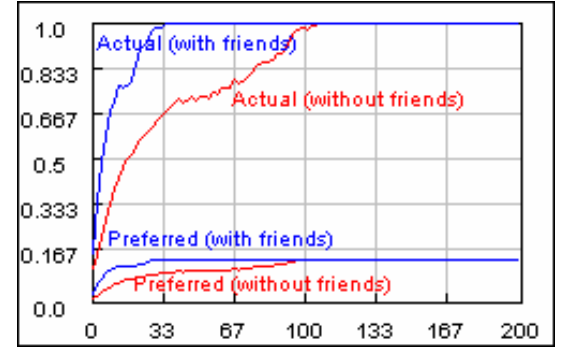

Fig. 1. (x: time in 1000s of cycles, y: actual affinities) Typical outcome for a modified homogeneous Axelrod model. Actual and preferred affinities with and without friends (partner selection).

Another experiment concerns a modified homogeneous Axelrod model with two initial populations where a cultural clash is expected, because each of the two populations occupies one half of the space and are separated by a linear border. Again, partner selection does not change the final outcome which is full monoculture in the population, but it speeds up a little the cultural contagion process, whereas additionally the average actual affinity before stabilization remains consistently higher than the no partner selection process. This is due to the border agents between the two populations that create fast individualistic partnerships.

A further experiment concerns a modified heterogeneous Axelrod model with an initially diverse population. Partner selection leads generally to the same but partial final affinities and is faster as well, as in previous cases (cf. Fig. 2).

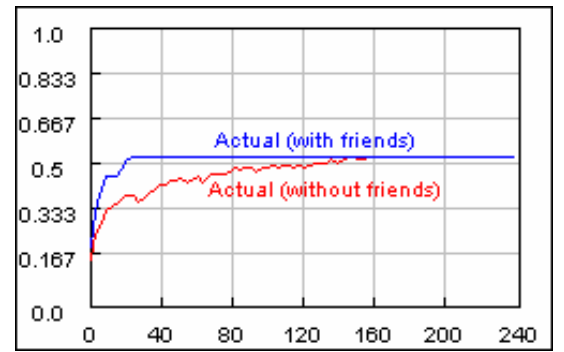

Fig. 2. (x: time in 1000s of cycles, y: actual affinities) Typical outcome for a modified heterogeneous Axelrod model. Actual affinities with and without friends (partner selection).

Additional experiments concern heterogeneous selfish, indifferent and complex models with an initially diverse population or with two initial populations where a cultural clash is expected. In the case of the mixed populations, as before, partner selection does not change the final outcomes and cultural convergence speeds up. The same applies to the case of two complex populations that meet, stabilization happens within a few cycles, because of the potential of agents to quickly build and maintain individualistic partnerships with some of their neighbors.

Overall, partner selection has been found not to alter substantially final expected outcomes without partner selection, but to markedly speed up cultural convergence because of the initial construction of partnerships that boost further development. From an evolutionary point of view, a partner selection mechanism would be promoted by evolution, because real biological and social systems generally do not have all the time needed to develop final, stable configurations, but operate in response to newly encountered situations. In such cases, it makes sense to dispose of means to find quick, working solutions to problems, and partner selection appears to be exactly such a means to quickly form small groups of high affinity, although it might be unstable in the longer term as further experiments show (in press).

\section{ATTRACTION}

\section{A. Rationale and Model}

Our motivation behind the introduction of an attraction mechanism is the general observation that in human societies, and especially in game-like and economic contexts, the agents' otherwise rational behavior can be heavily influenced by external psychological and social factors and also often it can be driven to behaviors outside their normal scope. By "external" we mean a factor or process that is not influenced itself by the primary agent task and does not normally participate in it. We are using the benchmark iterated prisoner's dilemma (IPD) [9][10] in its noisy version [11][12] as a study vehicle with a stronger bias toward defection, where we feel it could make sense to introduce such an external attraction factor. More specifically, we believe that biological evolution or, equivalently, social experience would spontaneously exploit any external factor that would induce better agent scores. This is particularly true for noisy IPD environments where agent scores may degrade abruptly, and especially when interactions are lengthier.

The attraction mechanism relies on our everyday experience that people tend to be good and cooperative with other people that attract them and tend to be "regular" with the rest. This translates in our model as:

$$
\begin{aligned}
& \text { If (attracted by the opponent) then play } \\
& \text { ALLC (always cooperate) } \\
& \text { Else play as usually (for example TFT) }
\end{aligned}
$$

We should note that noise is applied to the outcome of this behavior as well. We performed experiments with populations of agents playing a noisy IPD. The agents are interconnected via a "web of attraction" where each agent is connected to (attracted by) a number of others. The normal behavior of an agent is usually one of ALLC, ALLD (always defect), TFT (Tit-For-Tat) and Adaptive TFT [13], but we have also experimented occasionally with STFT (Suspicious TFT) or other strategies. We experimented with both uniform or mixed populations, whose agents have the same or diverse normal strategies. The reason we use mostly ALLC, ALLD, TFT and Adaptive TFT is that we want to make sure we explore the limits of our attraction mechanism by studying its effect on the extreme behaviors (ALLC and ALLD that act without feedback) as well as on the most intelligent ones (TFT that retaliates immediately and Adaptive TFT that tries to make sense of a situation). 
In previous work [13], we have classified usual IPD strategies in two categories: "retaliating" (or "rational") and "irrational". Retaliating strategies are those mostly TFTderived strategies that basically seek cooperation in the long run, but may start by exploring the opponent's reaction to a few initial D moves and will certainly retaliate the opponent's defections in some possibly intricate way (apparently, this general behavioral organization is the best choice so as to achieve maximum scores in the long run). For example, the suspicious tit-for-tat (or STFT) strategy starts by defecting, and then plays usual tit-for-tat. On the contrary, irrational strategies are those that do not employ any feedback from the game and play blindly using some innate law (although in some cases this can work, in the general case such strategies lose in the long run). For example, periodic strategies repeat patterns of C's and D's, such as CDD, CCD, CDCD etc. In this sense, ALLC and ALLD are irrational strategies whereas TFT and Adaptive TFT are retaliating ones. As in real life, we would expect irrational strategies to profit more from our attraction mechanism or other similar mechanisms and rational ones to be less dependent on such add-ons. In sum, we expect cooperation to be able to emerge in social interactions even in the absence of rationality and good reason.

We should stress the fact that the attraction mechanism described is in our own terms irrational in that it does not depend on any real feedback of the agent. Our results suggest that the coupling of reasoning mechanisms with reactive ones (such as attraction, physical, emotional, social or other) may be advantageous to social behavior and this is in line with current trends in cognitive and social science.

\section{B. Experiments}

In previous work [14] we have studied how the above attraction mechanism affects tournaments of agents interconnected via a "web of attraction". It is concluded there that the "social fitness", in terms of total score, profits from the introduction of the attraction mechanism, and especially for the irrational agents that are more vulnerable to still order in the absence of noise. Because each agent seeks to maximize its personal score and because this score depends crucially on its attraction relations with the other agents, it is reasonable to try to select partners. However, selection will be based solely on obtained scores between pairs of agents and not on explicitly perceived relations outside the interaction, i.e. the noisy IPD game. So, attraction affects interactions but cannot be directly perceived by the agents; hence, it is modeled as an uncontrollable (unconscious, at present) process.

We characterize our experiments by the number of agents $\mathrm{N}$ participating in a population, the attraction factor $\mathrm{M}$ (number of agents that an agent is attracted to), the partner set size $\mathrm{K}$ (number of agents that an agent interacts with) and the normal strategy of the agents. In each round, an agent selects K partners to interact with and receives a total score. The length of each noisy IPD game has been set to 100 cycles and the degree of noise to $10 \%$.
Partner selection uses the algorithm of section 2, instantiated as follows (the exact details of instantiation are highlighted):

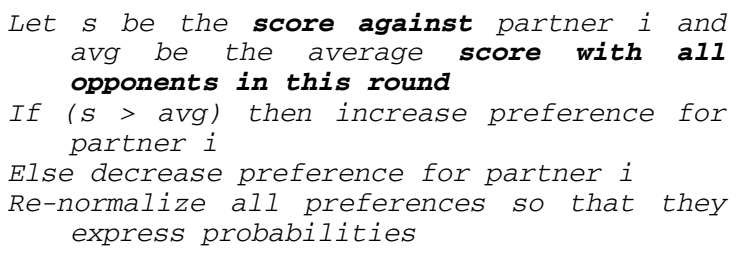

We give in Fig. 3 the results for the example case of a population of TFT agents $(\mathrm{N}=30, \mathrm{M}=5, \mathrm{~K}=5)$. Fig. 3 shows the evolution of the actual attraction factor, i.e. the average of the proportion of attracted partners per agent. This factor rises very quickly and converges toward the theoretically optimal value of 1 thanks to the partner selection mechanism. Moreover, the average score converges quickly toward its theoretical optimum of 300 (for a 100-cycle IPD game).

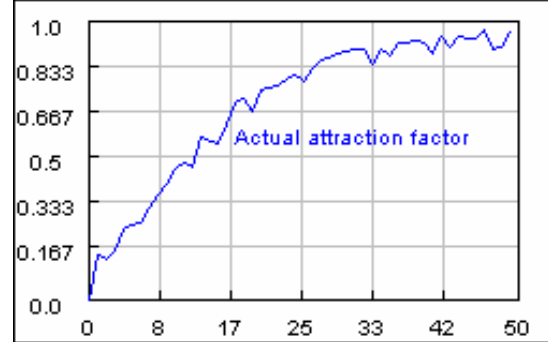

Fig. 3. Evolution of the actual attraction factor, i.e. the average of the proportion of attracted partners per agent: it approaches 1, which is the theoretical maximum.

Qualitatively similar results are obtained with uniform populations of other behavior and with mixed populations. The precise quantitative gains in average score remain however highly variable across different cases and depend on the actual behavior mix and on the attraction web.

\section{Cognitive Parameters of Partner SELEction}

The model of partner selection as defined and used in the preceding two behavioral contexts has a number of parameters whose effect on the final results obtained may be studied individually or in relation to one another. These are: the memory depth, the learning speed and the openness. As shown by their names, all three are of cognitive origin and may vary across individuals or cultural groups, i.e. they may be modeled and/or taken into account as both personality and group features.

\section{A. Memory Depth}

Because an agent selects partners to interact with, partners whose interactions are far behind in the past tend to or should have a discounted effect. This discount effect may be modeled in various ways. A direct way supported by our partner selection algorithm is to allow for an agent to interact simultaneously with $\mathrm{K}$ selected agents, so that the updating of the corresponding preferences will be synchronized and the effect of all previous interactions will be implicitly discounted. Fig. 4 shows comparative results in the cultural imitation case for $\mathrm{K}=0$ (no partner selection), $\mathrm{K}=1, \mathrm{~K}=2$ and 
$\mathrm{K}=3$ for the heterogeneous Axelrod model. It is shown that higher values of $\mathrm{K}$ speed up stabilization, at the expense of finding the theoretical optimum without partner selection. The homogeneous model leads to full final affinity for any value of $\mathrm{K}$ but faster for higher values of $\mathrm{K}$.

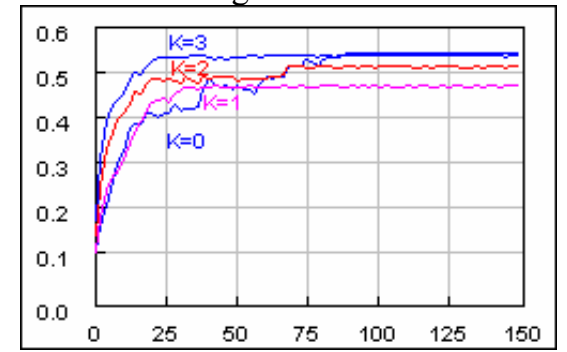

Fig. 4. (x: time in 500s of cycles, y: actual affinities) Outcomes for the modified heterogeneous Axelrod model. Actual affinities without partner selection (symbolized as $\mathrm{K}=0$ ) and with friends (partner selection) where $\mathrm{K}=1$ to 3 .

It has been verified with most of the personalized models of cultural imitation of section 3 that the final affinity levels reached deviate from the theoretical without partner selection as long as $\mathrm{K}$ is far from the theoretically necessary so as to have perfect information (here this limit is 3 or more, because all agents in a $2 \mathrm{D}$ grid have 8 neighbors except the agents that are positioned in the borders and corners that have 5 and 3 neighbors, respectively). It is shown elsewhere that higher values of $\mathrm{K}$ do not yield any more gain in stabilization speed.

\section{B. Learning Speed}

The preference updating (increase or decrease of probabilities) may vary in speed. More precisely, the increase and decrease of preference are modeled with the following formula, which is a variation of the law of effect:

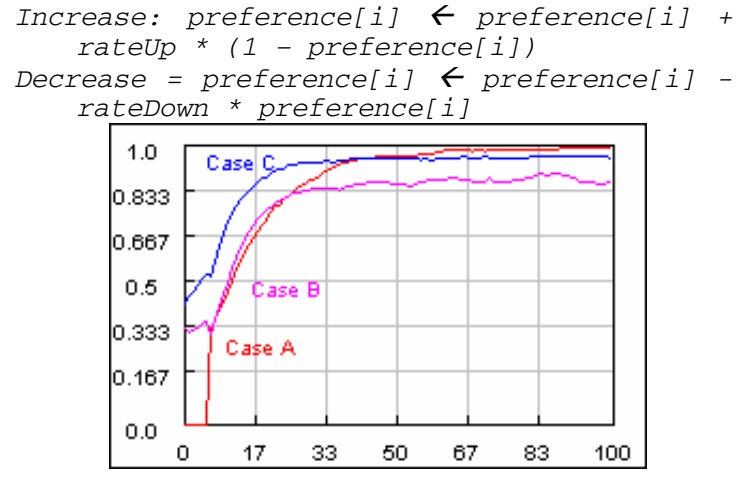

Fig. 5. Evolution of actual attraction factor for $\mathrm{N}=30, \mathrm{M}=5, \mathrm{~K}=5$, Case A: rate up $=0.1$ and rate down $=0.1$, Case B: rate up $=0.1$ and rate down $=0.5$, Case C: rate up $=0.5$ and rate down $=0.1$.

In all the other experiments reported, both learning rates are set to 0.1 . We may expect, though, that the exact values of these rates will influence the outcomes obtained, sometimes significantly. Fig. 5 shows comparative results in the attraction case for various combinations of rate values. These and other similar results show that "prudent" rather than "nervous" values of rates lead eventually to the best results and that these values should be balanced. However, the possibility of achieving faster but near optimal stabilization with an additional mechanism for regulating online the rate values cannot be ruled out.

\section{Openness}

Since the partner selection algorithm uses a probabilistic scheme, partners that have been associated with very low preferences will have almost no chance to be selected. This is not a problem as long as the behavioral environment does not change for any reason. If it does change, then an agent that used to be a non-preferred partner might become very attractive in the new environment. Thus the algorithm should have the potential to react to a changing environment and revert to a new preference set. To this end, the algorithm should allow a random partner to be selected from time to time instead of one according to the preference set. The probability of random choice in each cycle corresponds to an openness parameter that represents how often the partner selection algorithm will do exploration instead of exploitation.

As a demonstration in the attraction case, we have performed a perturbation study, equivalent to a regular invasion study performed in usual IPD games, where we reinitialize the attraction web after the social system has stabilized. For example, we run a system for 100 cycles until stabilization and then for another 100 rounds with a new attraction web. Fig. 6 presents comparative results for all three cases: the initial system (Case 1), the same system after attraction reinitialization (Case 2), and the second (reinitialized) system replayed with initial equiprobable preferences per agent (Case 3). It can be clearly seen that the (re)stabilization potential of such a social system depends on the initial conditions, that are the initial preferences of each agent. More specifically, the system when reinitialized after stabilization (Case 2) demonstrates a lower performance (inferior average score and attraction factor after stabilization), apparently due to the non-equiprobable initial partner preferences, unlike cases 1 and 3. This is both undesirable from a pure modeling perspective and biologically unrealistic: we would rather expect to see the system re-stabilize to the same final situation after any such perturbation.

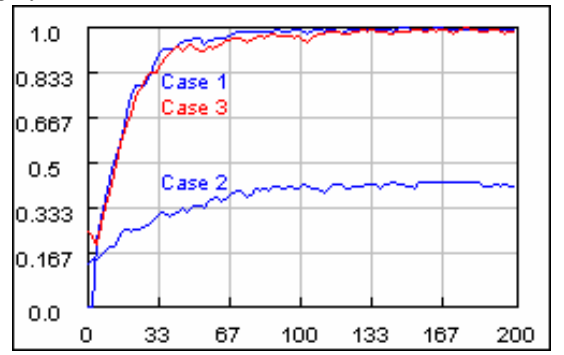

Fig. 6. Evolution of the actual attraction factor, i.e. the average of the proportion of attracted partners per agent in the three cases (see text). Case 2 cannot re-stabilize well enough, that is to the same near-optimal situations as the other two cases.

To face this requirement, we introduce an exploration factor as described above, i.e. as a probability with which an agent will choose a random partner for a particular 
interaction and not one based on its preference set. Because a system under stable attraction conditions does not need to restabilize, it is reasonable to assume that the exploration factor in this case should be 0 . To account for perturbations, however, we would need a nonzero value for this factor, say $0.08(8 \%)$. To integrate all the above requirements in one simple rule we use the following meta-regulation rule:

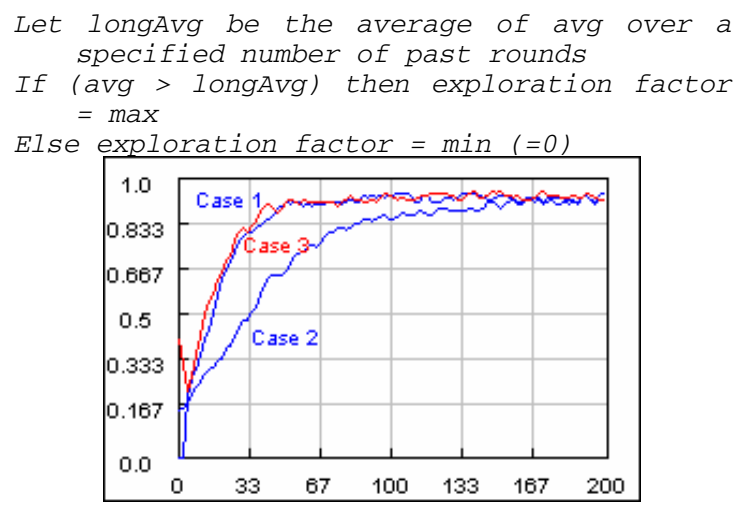

Fig. 7. Evolution of the actual attraction factor, i.e. the average of the proportion of attracted partners per agent in the three cases (see text). All cases lead to the same final stable situation.

With this additional rule the agents manage to regulate how much they actively search for new partners and for potential better opportunities. Fig. 7 gives the same results as fig. 6 , but this time with the additional meta-regulation rule. Note that the transitive results can be more variable and that the convergence speed is lower than with the previous rule: both these features are the price to pay for the occasional use of an exploration factor in partner selection.

We should note that many variants of the meta-regulation or openness management rule can be devised that will lead to the same qualitative results. Because this rule is so simple, the non-uniqueness of its exact details is an indication that an evolutionary process can actually discover it by trial-anderror. The same setup and rules can be used with variations of the attraction mechanism, for the cultural imitation case etc.

\section{DISCUSSION}

We have shown that, as theoretically expected, partner selection enhances immediate individual gains in behavioral social simulations. Partner selection allows fast local agent groups to emerge but these could prove detrimental in the longer term. The partner selection algorithm depends on a number of parameters of cognitive nature, namely memory depth, learning speed and openness. Memory depth has to be large enough to allow agents to find good enough partnerships; shallow memory is suboptimal. The learning speed has to be moderate and balanced between positive and negative events so to allow again good partnerships to emerge. Finally openness is mandatory in a changing environment so as to allow re-stabilization to new partnerships when necessary. These parameters should be taken into account through targeted studies when designing complex sociotechnical systems, such as social networking collaborative environments with human participants, so as to accommodate the diversity of the social and cultural background of participants. For example, all three parameters may have specific values due to cultural reasons, so that we may have social networking groups that are characterized by different triplets of values for (memory, speed, openness): one may be (deep, moderate, introvert) while another may be (shallow, fast, extrovert). It would be interesting and even imperative to investigate the effect of these values in the case of a cultural clash, i.e. an environment where two groups meet abruptly. It would be equally important to investigate what happens in a mixed but stable population when the external environments changes: it may be that one cultural group is more flexible than the other, or one is particularly resistant to change and so on. The factors influencing the partner selection process are therefore worth direct and deep investigation during the design and development of sociotechnical systems. Our next step toward a theoretical characterization of partner selection will be to use an abstract behavioral model and study comparatively all the parameters involved and their effects on the final structures that emerge.

\section{REFERENCES}

[1] Tesfatsion, L.: A trade network game with endogenous partner selection. In Advances in Computational Economics, H. Amman et al, Eds. Kluwer (1997)

[2] Tzafestas, E.S.: Emergence of social networks in systems with attraction. Proceedings Satellite Workshop Interacting Agents, Complexity and Interdisciplinary Applications (IACIA), European Conference on Complex Systems, Dresden (2007)

[3] Axelrod, A.: The dissemination of culture: A model with local convergence and global polarization. The Journal of Conflict Resolution, 41(2), 203-226 (1997)

[4] Shibanai, Y., Yasuno, S., Ishiguro, I.: Effects of global information feedback on diversity: Extensions to Axelrod's adaptive culture model. The Journal of Conflict Resolution, 45(1), 80-96 (2001)

[5] Trigg, A.B., Bertie, A.J., Himmelweit, S.F.: Modelling Bourdieu: An extension of the Axelrod cultural diffusion model". CRESC, Open University, Working Paper No. 49 (2008)

[6] Lanchier, N.: The Axelrod model for the dissemination of culture revisited. arXiv:1004.0365v1 (2010)

[7] Tzafestas, E.S.: Cultural diversity dynamics, Proceedings IEEE Symposium on Artificial Life, Paris (2011)

[8] Tzafestas, E.S.: Cultural dynamics, clashes and stability, Proceedings Indian Conference on Artificial Intelligence, Tumkur, India (2011)

[9] Axelrod, R., and Hamilton, W.D.: The evolution of cooperation, Science 211 1390-96 (1981)

[10] Axelrod, R.: The evolution of cooperation. Basic Books (1984)

[11] Kraines, D., and Kraines, V: Evolution of learning among Pavlov strategies in a competitive environment with noise, Journal of Conflict Resolution, 39(3):439-466 (1995)

[12] Molander, P.: The optimal level of generosity in a selfish, uncertain environment, Journal of Conflict Resolution, 31(4):692-724 (1987)

[13] Tzafestas, E.: Toward adaptive cooperative behavior, Proceedings of the Simulation of Adaptive Behavior Conference, Paris, Sept. (2000)

[14] Tzafestas, E.: Attraction and cooperation in noisy environments, Proceedings ESHIA (2007) 